\title{
Association of vitamin D deficiency with ventricular repolarization abnormalities
}

\author{
Denizhan Bagrul'1 , Fatih Atik ${ }^{2}$ \\ 1 Rize Recep Tayyip Erdogan University, Education and Research Hospital, Department of Pediatrics, Division of Pediatric Cardiology, Rize, Turkey \\ 2 Ankara Education and Research Hospital, Department of Pediatrics, Division of Pediatric Cardiology, Ankara, Turkey
}

\section{KEY WORDS}

sudden cardiac death, ventricular repolarization, vitamin $\mathrm{D}$ deficiency
Correspondence to: Denizhan Bagrul, MD, Department of Pediatrics and Division of Pediatric Cardiology, Faculty of Medicine, Recep Tayyip Erdogan University, Islampasa Mah., 53100 Rize,

Turkey, phone: +90 5079409399 , email: denizhanbagrul@hotmail.com Received: April 4, 2019. Revision accepted: July 3, 2019. Published online: July 3, 2019. Kardiol Pol. 2019; 77 (9): 853-858 doi:10.33963/KP.14888 Copyright by the Author(s), 2019

\begin{abstract}
BACKGROUND Vitamin D is involved in cardiac contractility and myocardial calcium hemostasis, and vitamin D deficiencies are known to cause various cardiovascular disorders and have been linked with sudden cardiac death.
\end{abstract}

AIMS The aim of the study was to evaluate repolarization distribution, represented by QT interval, corrected QT interval (QTc), QT dispersion, Tpeak-to-Tend (Tp-e) interval, Tp-e/QTc ratio, JT interval, JT dispersion, and Tp-e/JTpeak ratio in children with vitamin $\mathrm{D}$ deficiency. Moreover, we aimed to determine the relationship between ventricular repolarization anomalies and vitamin $D$ deficiency.

METHODS The study included 50 adolescent patients with vitamin D deficiency (vitamin $\mathrm{D}<20 \mathrm{ng} / \mathrm{ml}$ ), 50 adolescent patients with vitamin D insufficiency, and 50 age-matched controls (vitamin D level >30 ng/ml). QTc duration, QT dispersion, JTpeak duration, JT dispersion, Tp-e, Tp-e/JTpeak ratio, and Tp-e/ QTc ratio were recorded on electrocardiogram.

RESULTS Patients with vitamin D deficiency or insufficiency had longer Tp-e interval $(P<0.001)$, while Tp-e/QTc and Tp-e/JTpeak ratios were found to be increased in the same group of patients $(P=0.005$ and $P<0.001$, respectively). QT dispersion and JT dispersion were higher in the deficient group when compared with the other groups ( $P=0.045$ and $P=0.02$, respectively).

CONCLUSIONS The present study, conducted in a pediatric population, is the first in the current literature to assess the relationship between ventricular repolarization anomalies and vitamin D deficiency.

INTRODUCTION Vitamin D and its receptor (VDR) play an important role in a broad range of organ functions, and it has been shown that there is a significant presence of VDR in the cardiac muscle. ${ }^{1,2}$ Vitamin D affects cardiac contractility and myocardial calcium hemostasis, ${ }^{3}$ and vitamin D deficiency has been shown to cause various cardiovascular disorders, including hypertension, coronary artery disease, heart failure, and peripheric vascular disease. ${ }^{4}$ Vitamin D deficiency has also been associated with ionic channel disorders and autonomic function disorder, which may cause fatal cardiac arrhythmia and sudden cardiac death (SCD). 5,6

Ventricular repolarization (VR) anomalies identified on electrocardiography (ECG) may predict malignant ventricular arrhythmia. To date, QT interval, corrected QT interval (QTc), and QT dispersion (QTd) have been used for the detection of VR anomalies. ${ }^{7}$ In addition to these, in recent years, it has been reported that new indices such as Tpeak-Tend (Tp-e), Tp-e/QTc, JTpeak (JTp) interval, corrected JT (JTc) distance, JT dispersion (JTd), and Tp-e/JTp ratio can indicate the existence of VR anomalies. ${ }^{8-12}$

The present study evaluates repolarization distribution, represented by QT interval, QTc, QTd, Tp-e interval, Tp-e/QTc ratio, JTp interval, JTd, and Tp-e/JTp ratio in children with vitamin $\mathrm{D}$ deficiency, and determines the relationship between VR anomalies and vitamin D deficiency. 


\section{WHAT'S NEW?}

Vitamin D deficiencies can lead to various cardiovascular disorders, including hypertension, coronary artery disease, heart failure, and sudden cardiac death. The most common cause of sudden cardiac death is ventricular arrhythmia. The present study demonstrates that Tpeak-to-Tend (Tp-e) interval, Tp-e/ corrected QT ratio, Tp-e/JTpeak ratio, QT dispersion, and JT dispersion, as potentially new repolarization markers for malignant ventricular arrhythmia, were increased in people with vitamin $D$ deficiencies or insufficiencies. These findings support the association between vitamin $D$ deficiency and the risk of sudden cardiac death reported in previous studies. To the best of our knowledge, there have been no studies to date using these new repolarization indices, Tp-e interval, Tp-e/corrected QT ratio, Tp-e/JTpeak ratio, and JT dispersion, in patients with vitamin $D$ deficiency.

METHODS The study was conducted from September 2018 to January 2019 in the pediatric cardiology division of a tertiary center. A total of 150 patients aged from 10 to 18 years were included in the study. Out of those, 50 patients with vitamin D levels of less than $20 \mathrm{ng} / \mathrm{ml}$ formed group $1 ; 50$ patients with vitamin D levels of 21 to $29 \mathrm{ng} / \mathrm{ml}$ formed group 2; and 50 patients with vitamin D levels of more than $30 \mathrm{ng} / \mathrm{ml}$ formed group 3 .
All patients underwent transthoracic echocardiography using a Vivid S5 (GE Vingmed Ultrasound AS, Horten, Norway) to exclude structural heart disease. The exclusion criteria for the study were: younger than 10 years or older than 18 years of age; structural cardiac disease; dysrhythmia; U wave on ECG; chronic disease related to the gastrointestinal, renal, or noncardiac system; continuous use of medication; morbid obesity or metabolic syndrome; and acute infection. None of the participants were taking vitamin D supplements.

Serum 25-hydroxyvitamin D (25[OH]D) levels were measured using ADVIA Centaur and ADVIA Centaur XP systems (Siemens Healthcare Diagnostics, Deerfield, Illinois, United States). Blood samples were collected between $8 \mathrm{AM}$ and $10 \mathrm{AM}$ after a fast of at least $8 \mathrm{~h}$.

A standard 12-lead ECG was used to evaluate the Tp-e interval, QT interval, QTc interval, JTc interval, JTp interval, QTd, JTd, Tp-e/QTc ratio, and Tp-e/JTp ratio. The ECG was performed after 10 minutes of rest in a supine position at $25 \mathrm{~mm} / \mathrm{s}$ paper speed and at $10 \mathrm{mV} / \mathrm{mm}$ amplitude, using the same digital ECG device (EDAN SE-1010 PC ECG system, EDAN Instruments, Inc.,

TABLE 1 Baseline demographic, clinical, and echocardiographic characteristics of the 3 study groups

\begin{tabular}{|c|c|c|c|c|c|}
\hline \multicolumn{2}{|l|}{ Parameter } & $\begin{array}{l}\text { Group } 1 \\
\text { Deficient vitamin D status } \\
(n=50)\end{array}$ & $\begin{array}{l}\text { Group } 2 \\
\text { Insufficient vitamin D } \\
\text { status }(n=50)\end{array}$ & $\begin{array}{l}\text { Group } 3 \\
\text { Sufficient vitamin D } \\
\text { status }(n=50)\end{array}$ & Pvalue \\
\hline \multicolumn{6}{|c|}{ Baseline demographic or clinical parameter } \\
\hline Age, y & & $14.8(3.8)$ & $14.1(3.6)$ & $14.2(3.8)$ & 0.76 \\
\hline \multirow[t]{2}{*}{ Sex, n (\%) } & Male & $26(52)$ & $25(50)$ & $25(50)$ & \multirow[t]{2}{*}{0.97} \\
\hline & Female & $24(48)$ & $25(50)$ & $25(50)$ & \\
\hline Body mass index, $\mathrm{kg} / \mathrm{m}^{2}$ & & $20.6(2.3)$ & $20.3(2.5)$ & $20.1(2.3)$ & 0.84 \\
\hline \multirow[t]{2}{*}{ Blood pressure, mm Hg } & $\begin{array}{l}\text { Systolic blood } \\
\text { pressure }\end{array}$ & $112.2(10.2)$ & $109.8(10.5)$ & $108.1(9.8)$ & 0.69 \\
\hline & $\begin{array}{l}\text { Diastolic blood } \\
\text { pressure }\end{array}$ & $66.6(7.9)$ & $64.0(8.2)$ & $63(8.5)$ & 0.46 \\
\hline \multicolumn{6}{|c|}{ Baseline echocardiographic parameter } \\
\hline LVEF, \% & & $68.1(5.7)$ & $69.1(7.2)$ & $68.4(6.4)$ & 0.83 \\
\hline $\mathrm{FS}, \%$ & & $38.2(4.1)$ & $39.2(3.8)$ & $38.0(4.6)$ & 0.36 \\
\hline LVEDD, mm & & $40.7(4.8)$ & $41.4(6.0)$ & $41.1(6.9)$ & 0.4 \\
\hline LVESD, mm & & $24.4(3.3)$ & $25.3(4.0)$ & $24.6(3.8)$ & 0.54 \\
\hline $\mathrm{LAD}, \mathrm{mm}$ & & $24.2(2.4)$ & $25(2.7)$ & $24.4(3.2)$ & 0.78 \\
\hline LVMPI & & $0.33(0.04)$ & $0.32(0.03)$ & $0.34(0.08)$ & 0.66 \\
\hline TAPSE, mm & & $23.0(3.8)$ & $23.6(3.4)$ & $23.5(4.5)$ & 0.82 \\
\hline Mitral E, m/s & & $1.72(0.55)$ & $1.82(0.4)$ & $1.77(0.67)$ & 0.2 \\
\hline Mitral A, m/s & & $1.05(0.38)$ & $1.1(0.41)$ & $1.08(0.37)$ & 0.11 \\
\hline Mitral E/A, m/s & & $1.51(0.34)$ & $1.54(0.56)$ & $1.59(0.44)$ & 0.18 \\
\hline
\end{tabular}

One-way analysis of vairance ( $P<0.05$ is considered significant). Data are presented as mean (SD) unless otherwise indicated.

Abbreviations: LVEF, left ventricular ejection fraction; FS, fractional shortening; LVEDD, left ventricle end-diastolic diameter; LVESD, left ventricle end-systolic diameter; LVMPI, left ventricular myocardial performance index; TAPSE, tricuspid annular plane systolic excursion 
Shenzhen, China) in each patient. The measurements were obtained from magnified ECG results by 2 pediatric cardiologists who were blinded to the vitamin D levels of the participants. The QT range was measured from the start of the QRS complex to the end of the T wave, and was corrected using the Bazett formula for heart rate. The Tp-e interval was defined as the range between the peak of the $\mathrm{T}$ wave to the end of the $\mathrm{T}$ wave. The Tp-e interval measurement was made from precordial derivations. JTp was defined as the interval from the $\mathrm{J}$ point to the peak of the $\mathrm{T}$ wave. The JTc interval was calculated by subtracting the QRS duration from the QTc interval, and the JTd was defined as the difference between the maximum and minimum JT interval averages. QTd was defined as the maximum difference between the QT intervals of 2 leads of the 12-lead ECG, and the Tp-e/QTc and Tp-e/JTp ratios were calculated from these measurements. Intraobserver and interobserver variations for Tp-e interval, QT distance, and JTp interval measurements were less than $5 \%$ (intraobserver variability $2.5 \%$, $3.8 \%$, and $3.4 \%$, respectively, and interobserver variability $2.9 \%, 3.9 \%$, and $3.6 \%$, respectively).

Statistical analysis Data were analyzed using the SPSS Statistics for Windows software package, Version 22.0 (IBM Corp., Armonk,
New York, United States). Continuous variables with abnormal distribution were log transformed prior to analysis. Qualitative variables were presented as number (percentage), while continuous variables were expressed as mean (SD). The differences in the mean levels of the continuous variables between patients with sufficient, insufficient, and deficient levels of vitamin D were determined with a 1-way analysis of variance (ANOVA), and with the Tukey post-hoc test for multiple comparisons. A $P$ value of less than 0.05 was considered significant.

The study protocol was approved by the local ethics committee, and informed consent was obtained prior to the study from all patients or their families.

RESULTS The baseline demographic characteristics of the 3 groups are presented in TABLE 1 . The groups were compared for age, gender, height, weight, body mass index, and systolic and diastolic blood pressure, and no differences were found between the groups.

Measurements of the left and right ventricular diameters, left atrium diameter, left ventricular inflow features, left ventricular ejection fraction, shortening fraction, myocardial

TABLE 2 Electrocardiographic characteristics of the 3 study groups

\begin{tabular}{|c|c|c|c|c|}
\hline Electrocardiographic parameter & $\begin{array}{l}\text { Group } 1 \\
\text { Deficient vitamin D } \\
\text { status }(n=50)\end{array}$ & $\begin{array}{l}\text { Group } 2 \\
\text { Insufficient vitamin D } \\
\text { status }(n=50)\end{array}$ & $\begin{array}{l}\text { Group } 3 \\
\text { Sufficient vitamin D } \\
\text { status }(n=50)\end{array}$ & $P$ value \\
\hline Heart rate, bpm & $88.13(13.74)$ & $90.13(14.56)$ & $88.25(13.25)$ & 0.84 \\
\hline QRS, ms & $81.1(14.5)$ & $82.4(15.4)$ & $82.5(15.3)$ & 0.94 \\
\hline QT, ms & $378(25)$ & $377(21)$ & $372(30)$ & 0.26 \\
\hline QTc, ms & $398(28)$ & $396(30)$ & $392(38)$ & 0.19 \\
\hline JTc, ms & $315.4(19.2)$ & $312(22.1)$ & $308.09(25.09)$ & 0.1 \\
\hline Tp-e, ms & $82.2(8.8)^{\mathrm{a}}$ & $82.0(8.6)^{b}$ & $70.4(10.4)^{\mathrm{a}, \mathrm{b}}$ & $<0.001$ \\
\hline Tp-ec, ms & $90.9(8.6)^{\mathrm{a}}$ & $92.4(12.4)^{b}$ & $77.2(10.5)^{\mathrm{a}, \mathrm{b}}$ & $<0.001$ \\
\hline JTp, ms & $228(16)$ & $224(15)$ & $219(22)$ & 0.11 \\
\hline JTend, ms & $280(22)$ & $278(16)$ & $270(18)$ & 0.09 \\
\hline Tp-e/QTc & $0.21(0.02)^{\mathrm{a}}$ & $0.21(0.02)^{b}$ & $0.17(0.02)^{\mathrm{a}, \mathrm{b}}$ & 0.005 \\
\hline Tp-e/JTp & $0.38(0.05)^{\mathrm{a}}$ & $0.36(0.04)^{\mathrm{b}}$ & $0.3(0.07)^{a, b}$ & $<0.001$ \\
\hline QTd, ms & $64(5.2)^{a, c}$ & $57.3(4.6)^{c}$ & $56.1(4.4)^{\mathrm{a}}$ & 0.045 \\
\hline JTd, ms & $28.7(11.2)^{\mathrm{a}, \mathrm{c}}$ & $21.8(10.2)^{c}$ & $20.52(9.3)^{\mathrm{a}}$ & 0.02 \\
\hline
\end{tabular}

One-way analysis of variance ( $P<0.05$ is considered significant). Data are presented as mean (SD).

a The post hoc analysis revealed a difference between group 1 and group 3.

b The post hoc analysis revealed a difference between group 2 and group 3 .

c The post hoc analysis revealed a difference between group 1 and group 2 .

Abbreviations: Bpm, beats per minute; QTc, corrected QT interval; JTc, corrected JT interval; JTp, JTpeak; Tp-e, Tpeak-to-Tend interval; Tp-ec, corrected Tend-to-Tpeak interval; QTd, QT dispersion; JTd, JT dispersion 
performance index, and tricuspid annular plane systolic excursion were made on transthoracic echocardiography and no differences were found between the groups (TABLE 1).

The ECG characteristics of the groups, including heart rate, QT, QTc, Tp-e interval, Tp-e/QTc ratio, JTp interval, Tp-e/JTp ratio, JTd, and QTd, are presented in TABLE2. The heart rates were similar between the groups. The mean (SD) Tp-e intervals in groups 1 and 2 were 82.2 (8.8) ms and 82.0 (8.6) ms, respectively. However, in group 3, the Tp-e interval was found to be longer than in groups 1 and 2 (mean [SD], 70.4 [10.4] ms) $(P<0.001)$. The mean (SD) Tp-e/QTc ratio in groups 1 and 2 was $0.22(0.03)$ and 0.21 (0.03), respectively, and was considerably higher in group 3 (mean [SD], 0.28 [0.05]) $(P=0.005)$. The mean (SD) Tp-e/JTp ratio in groups 1 and 2 was 0.38 (0.05) and 0.36 (0.04), respectively, while in group 3 the mean ratio was found to be higher (mean [SD], 0.3 [0.07]) $(P<0.001)$. QTd and JTd were found to be higher in group 1 than in the other groups (mean [SD], 64 [5.2] ms; $P=$ 0.045 ; and 28.7 [11.2] ms; $P=0.02$, respectively). No differences were identified in the QTc and JTc intervals between the groups; and no differences were detected between groups 1 and 2 in any parameters other than QTd and JTd times.

DISCUSSION It was found that Tp-e interval, Tp-e/QTc and Tp-e/JTp ratios, QTd, and JTd, as potentially new repolarization markers for malignant ventricular arrhythmia, were increased in those with vitamin D deficiency or insufficiency. These findings support the association between vitamin D deficiency and the risk of SCD reported in previous studies. ${ }^{5,6,13}$ To the best of our knowledge there have been no studies to date using the new repolarization indices, Tp-e interval, Tp-e/QTc ratio, Tp-e/JTp ratio, and JTd, in patients with vitamin $\mathrm{D}$ deficiency.

An adequate vitamin $D$ intake is necessary for the optimal functioning of many tissues and organs as well as for bone health. Vitamin D receptors can be found in many tissues and cells, including enterocytes, osteoblasts, pancreatic $\beta$ cells, vascular endothelial cells, parathyroid gland cells, the liver, the immune system, and renal tubule cells. The active form of vitamin $\mathrm{D}$ (1,25-hydroxyvitamin D) binds to VDRs, and this complex, along with some other factors, changes the gene expression by binding to deoxynucleic acid. Recent studies have also identified VDR in the cardiac muscle. ${ }^{4,14}$ Epidemiologic studies have linked low 25(OH)D levels with coronary risk factors and adverse cardiovascular events, ${ }^{6,15}$ and vitamin $\mathrm{D}$ deficiency has also been linked to hypertension, diabetes and metabolic syndrome, hyperlipidemia, atherosclerosis, carotid intima-media thickening, left ventricular hypertrophy, and congestive heart disease. ${ }^{1}$
Vitamin D deficiency has also been linked with ionic channel disorders and autonomic function disorder, which can cause SCD and malignant cardiac arrhythmia, as well as many other adverse cardiovascular effects. ${ }^{16,17}$ It is also thought that a disruption in the synthesis of vitamin D and parathyroid hormone will result in structural and ionic channel reshaping, which may increase disposition to cardiac arrhythmia. Forman et $\mathrm{ll}^{18}$ reported that patients with the lowest vitamin D levels had the highest levels of angiotensin II, and that renin-angiotensin-aldosterone system was abnormally active in the absence of vitamin D. It is known empirically that vitamin $\mathrm{D}$ deficiencies or disruptions in receptors induce the activation of renin-angiotensin-aldosterone system and that this leads to the activation of mineralocorticoid receptor, an increase in the flow in the calcium channel towards the cell, and a decrease in potassium flow outside the cell. ${ }^{19,20}$ These changes may, in time, lengthen the repolarization range and cardiac axion potential, and can increase sensitivity to malign ventricular arrhythmias.

Pilz et al ${ }^{15}$ followed a total of 3299 patients who were referred for a routine coronary angiography for a mean of 7.7 years and investigated fatal cardiac events. An increased risk of SCD was determined in those with severe vitamin D deficiencies (hazard ratio [HR], 5.05; 95\% confidence interval [CI], 2.13-11.97). In a study conducted in Germany involving 1108 patients with diabetes undergoing hemodialysis, it was reported that those with vitamin $\mathrm{D}$ deficiency were 3-fold more likely to suffer cardiac death when compared with those with sufficient vitamin D (HR, 2.99; 95\% CI, 1.39-6.40), and it was concluded that severe vitamin D deficiency is strongly associated with SCD. ${ }^{13}$ Deo et $\mathrm{al}^{5}$ followed 2312 elderly participants for a mean of 14 years to investigate the relationship between vitamin D levels and SCD. It was reported that vitamin $\mathrm{D}$ deficiency and high parathormone levels were a strong marker of SCD risk (HR, 2.19; $95 \%$ CI $1.17-4.10$ ), and it was thus recommended that further studies be conducted to investigate the relationship between arrhythmogenic events and vitamin D metabolism. ${ }^{5}$

The most common cause of SCD is ventricular arrhythmia. ${ }^{21}$ However, VR is a complex electric event that is a critical step in the development of ventricular arrhythmia. The use of indices that reveal repolarization anomalies on ECG is therefore important in determining ventricular arrhythmia events and the risk of SCD. Abnormal QT interval, QTc, and QTd values, which have been used traditionally as myocardial repolarization markers, indicate an increased risk of ventricular arrhythmia. ${ }^{22,23}$ No relationship could be identified between vitamin D levels and QT and QTc times in the present study, although QTd and JTd were increased in those with vitamin 
$\mathrm{D}$ deficiency. Yetkin et al, ${ }^{24}$ in a study on patients with type 2 diabetes, reported that QT interval and QTd were increased in those with vitamin D deficiency as compared with controls (434 vs 423 ms; $P<0.001$; and 53.8 vs $43.2 \mathrm{~ms}$; $P<0.001$, respectively). Similarly, Kim et al, ${ }^{25}$ in their study in patients undergoing hemodialysis, detected decreases in QT distance and QTd without hemodynamic or biochemical changes with 1,25-hydroxyvitamin $\mathrm{D}$. The authors conclude that an active vitamin D metabolite has a cardioprotective effect, resulting from the increase in the levels of intracellular calcium in cardiac muscle cells, and an indirect effect on the reduction of the QTd through the regression of the left ventricular hypertrophy.

In recent years, new indices that are considered to be more sensitive in various clinical settings for the evaluation of VR have been adopted. Tp-e interval is a relatively new ECG parameter that has been proposed for the measurement of global dispersion of repolarization. ${ }^{8}$ It has been demonstrated that $\mathrm{Tp}$ coincides with the earliest end of repolarization, whereas $\mathrm{Te}$ coincides with the latest end of repolarization. ${ }^{12}$ Electrophysiological studies have revealed a relationship between a long Tp-e interval and ventricular arrhythmia, ${ }^{26}$ while the JTp interval is a more specific repolarization marker, in that the QT range represents depolarization and repolarization, and is affected by QRS time. ${ }^{12}$ Furthermore, the Tp-e/QTc ratio and Tp-e/JTp ratio can be seen as more sensitive indexes for arrhythmogenesis when compared with Tp-e interval or QT interval used alone. ${ }^{27,28}$

It can be concluded that the effects of vitamin D on the cardiac autonomic neural system (ANS) lead to VR disorders. Vitamin D regulates some mechanisms, including inflammatory signalization and neurotransmitter synthesis in the central neural system that regulates cardiovascular activity. Furthermore, cardiac ANS dysfunction has been identified as an independent predictor of sudden cardiac mortality. ${ }^{29,30}$ The midbrain and brainstem, in which several neurons for the ANS are located, have been shown to contain high concentrations of vitamin $\mathrm{D}$ receptors, resulting in vitamin $\mathrm{D}$ being actively taken up in these high central control centers, given its specific role in ANS regulation. ${ }^{31,32}$ Mann et $\mathrm{al}^{33}$ noted that increased vitamin D levels in healthy people are associated with significant improvements in cardiac ANS balance, given its enhancement of cardioprotective vagal activity in response to external stresses. In addition, it was shown that vitamin $\mathrm{D}$ deficiency was an important regulator in pathways related to epilepsy and spinal injury in neurocardiovascular disease. ${ }^{34,35}$ When considered together, these data demonstrate that vitamin $\mathrm{D}$ deficiency affects mortality risk due to CVD by mediating the activities of ANS.
Another important point is related to levels of vitamin D in the blood. Most experts define vitamin $\mathrm{D}$ deficiency as a $25(\mathrm{OH}) \mathrm{D}$ level of $<20 \mathrm{ng} / \mathrm{ml}$, at which point, it is argued, clinical findings occur. It is thus stated that the desired vitamin D level is at least $20 \mathrm{ng} / \mathrm{ml},{ }^{36,37} \mathrm{al}-$ though in the present study it was determined that cardiac effects also occurred in individuals with insufficient $(21-29 \mathrm{ng} / \mathrm{ml})$ serum vitamin D levels. Accordingly, clinicians must consider this when determining the vitamin $\mathrm{D}$ levels of their patients.

Conclusions The present study, conducted in a pediatric population, is the first to reveal the relationship between VR anomalies and vitamin $\mathrm{D}$ deficiency. We demonstrated that Tp-e interval, Tp-e/QTc ratio, Tp-e/JTp ratio, JTd, and QTd are significantly increased in people with vitamin D deficiency or insufficiency, which did not corroborate the results of previous studies associating vitamin $\mathrm{D}$ deficiencies with an increased risk of sudden cardiac mortality. Clinicians should consider these findings when following the vitamin D levels of their patients. Further studies involving bigger samples are warranted to investigate the relationship between vitamin D and cardiac malignant arrhythmia mechanisms.

\section{ARTICLE INFORMATION}

ACKNOWLEDGMENTS This research did not receive any specific grant from funding agencies in the public, commercial, or not-for-profit sectors. CONFLICT OF INTEREST None declared.

OPEN ACCESS This is an Open Access article distributed under the terms of the Creative Commons Attribution-NonCommercial-NoDerivatives 4.0 International License (CC BY-NC-ND 4.0), allowing third parties to download articles and share them with others, provided the original work is properly cited, not changed in any way, distributed under the same license, and used for noncommercial purposes only. For commercial use, please contact the journal office at kardiologiapolska@ptkardio.pl.

HOW TO CITE Bagrul D, AtikF. Association of vitamin D deficiency with ventricular repolarization abnormalities. Kardiol Pol. 2019; 77: 853-858. doi:10.33963/KP.14888

\section{REFERENCES}

1 Vanga SR, Good M, Howard PA, et al. Role of vitamin D in cardiovascular health. Am J Card. 2010; 106: 798-805.

2 Anderson JL, May HT, Horne BD, et al. Relation of vitamin D deficiency to cardiovascular risk factors, disease status, and incident events in a general healthcare population. Am J Card. 2010; 106: 963-968.

3 Al Mheid I, Patel RS, Tangpricha V, et al. Vitamin D and cardiovascular disease: is the evidence solid? Eur Heart J. 2013; 34: 3691-3698.

4 Nibbelink KA, Tishkoff DX, Hershey SD, et al. 1,25(OH)2-vitamin D3 actions on cell proliferation, size, gene expression, and receptor localization, in the HL-1 cardiac myocyte. J Steroid Biochem Mol Biol. 2007; 103: 533-537.

5 Deo R, Katz R, Shlipak MG, et al. Vitamin D, parathyroid hormone and sudden cardiac death: results from the cardiovascular health study. Hypertension. 2011; 58: 1021-1028.

6 Wang TJ, Pencina MJ, Booth SL, et al. Vitamin D deficiency and risk of cardiovascular disease. Circulation. 2008; 117: 503-511.

7 Monitillo F, Leone M, Rizzo C, et al. Ventricular repolarization measures for arrhythmic risk stratification. World J Cardiol. 2016; 8: 57-73.

8 Antzelevitch C, Sicouri S, Di Diego JM, et al. Does Tpeak-Tend provide an index of transmural dispersion of repolarization? Heart Rhythm. 2007; 4: 1114-1116.

9 Karaman K, Altunkaş F, Cetin M, et al. New markers for ventricular repolarization in coronary slow flow: Tp-e interval, Tp-e/QT ratio, and Tp-e/QTc ratio. Ann Noninvasive Electrocardiol. 2015; 20: 338-344.

10 Crow RS, Hannan PJ, Folsom AR. Prognostic significance of corrected QT and corrected JT interval for incident coronary heart disease in a general population 
sample stratified by presence or absence of wide QRS complex: the ARIC study with 13 years of follow-up. Circulation. 2003; 108: 1985-1989.

11 Coşkun FY, Elboğa G, Altunbaş G, et al. Evaluation of ventricular repolarization features with Tp-e, Tp-e/QTc, JTc and JTd during electroconvulsive therapy. J Electrocardiol. 2018; 51: 440-442.

12 Tse G, Yan BP. Traditional and novel electrocardiographic conduction and repolarization markers of sudden cardiac death. EP Europace. 2017; 19: 712-721.

13 Drechsler C, Pilz S, Obermayer-Pietsch B, et al. Vitamin D deficiency is associated with sudden cardiac death, combined cardiovascular events, and mortality in haemodialysis patients. Eur Heart J. 2010; 31: 2253-2261.

14 Tishkoff DX, Nibbelink KA, Holmberg KH, et al. Functional vitamin D receptor (VDR) in the t-tubules of cardiac myocytes: VDR knockout cardiomyocyte contractility. Endocrinology. 2007; 149: 558-564.

15 Pilz S, März W, Wellnitz B, et al. Association of vitamin D deficiency with heart failure and sudden cardiac death in a large cross-sectional study of patients referred for coronary angiography. J Clin Endocrinol Metab. 2008; 93: 3927-3935.

16 Bolland MJ, Grey A, Avenell GD, et al. Calcium supplements with or without vitamin D and risk of cardiovascular events: reanalysis of the Women's Health Initiative limited access dataset and meta-analysis. BMJ. 2011; 342:d2040.

17 Mann MC, Hollenberg MD, Hanley DA, et al. Vitamin D, the autonomic nervous system, and cardiovascular risk. Physiol Rep. 2015; 3: pii:e12349.

18 Forman JP, Giovannucci E, Holmes MD, et al. Plasma 25-hydroxyvitamin D levels and risk of incident hypertension. Hypertension. 2007; 49: 1063-1069.

19 Li YC, Kong J, Wei M, et al. 1,25- Dihydroxyvitamin D(3) is a negative endocrine regulator of the renin-angiotensin system. J Clin Invest. 2002; 110: 229-238.

20 Perrier E, Kerfant BG, Lalevee N, et al. Mineralocorticoid receptor antagonism prevents the electrical remodeling that precedes cellular hypertrophy after myocardial infarction. Circulation. 2004; 110: 776-783.

21 Kannel WB, Plehn JF, Cupples LA. Cardiac failure and sudden death in the Framingham Study. Am Heart J. 1988; 115: 869-875.

22 Schouten EG, Dekker JM, Meppelink $P$, et al. QT interval prolongation predicts cardiovascular mortality in an apparently healthy population. Circulation. 1991; 84: 1516- 1523.

23 Moss Al. Measurement of the QT interval and the risk associated with QT(c) interval prolongation a review. Am J Cardiol. 1993; 72: 23-25.

24 Yetkin D0, Kucukkaya B, Turhan M, et al. The effect of 25-hydroxyvitamin $\mathrm{D}$ levels on QT interval duration and dispersion in type 2 diabetic patients. Croat Med J. 2015; 56: 525-530.

25 Kim HW, Park CW, Shin YS, et al. Calcitriol regresses cardiac hypertrophy and QT dispersion in secondary hyperparathyroidism on hemodialysis. Nephron Clin Pract. 2006; 102: 21-29.

26 Smetana P, Schmidt A, Zabel M, et al. Assessment of repolarization heterogeneity for prediction of mortality in cardiovascular disease: peak to the end of the T wave interval and nondipolar repolarization components. J Electrocardiol. 2011; 44: 301-308.

27 Gupta P, Patel C, Patel H, et al. Tpe/QT ratio as an index of arrhythmogenesis. J Electrocardiol. 2008; 41: 567-574.

28 Alvarado-Serrano C, Ramos-Castro J, Palla's-Areny R. Novel indices of ventricular repolarization to screen post myocardial infarction patients. Comput Biol Med. 2006; 36: 507-515

29 Garcion E, Wion-Barbot N, Montero-Menei CN, et al. New clues about vitamin D functions in the nervous system. Trends Endocrinol Metabolism. 2002; 13: 100-105. 30 DeLuca GC, Kimball SM, Kolasinski ], et al. The role of vitamin D in nervous system health and disease. Neuropathol Appl Neurobiol. 2013; 39: 458-484

31 Tsuji H, Venditti Jr Fl, Manders ES, et al. Reduced heart rate variability and mortality risk in an elderly cohort. The Framingham Heart Study. Circulation. 1994; 90: 878-883.

32 Thayer JF, Yamamoto SS, Brosschot JF. The relationship of autonomic imbalance, heart rate variability, and cardiovascular disease risk factors. Int I Cardiol. 2010; 141: 122-131.

33 Mann MC, Exner DV, Hemmelgarn BR, et al. Vitamin D supplementation is associated with improved modulation of cardiac autonomic tone in healthy humans. J Int Cardiol. 2014; 172: 506-508.

34 Scorza FA, Albuquerque MD, Arida RM, et al. Benefits of sunlight: vitamin $D$ deficiency might increase the risk of sudden unexpected death in epilepsy. Med Hypotheses. 2010; 7: 158-161.

35 Zebracki K, Hwang M, Patt PL, et al. Autonomic cardiovascular dysfunction and vitamin D deficiency in pediatric spinal cord injury. J Ped Rehab Med. 2013; 6: 45-52.

36 Holick MF. Vitamin D Deficiency. N Engl J Med. 2007; 357: 266-281.

37 Holick MF, Binkley NC, Bischoff-Ferrari HA, et al. Evaluation, treatment, and prevention of vitamin D deficiency: an Endocrine Society clinical practice guideline. J Clin Endocrinol Metab. 2011; 96: 1911-1930. 